\title{
QoS Oriented Multiobjective Optimizer for Radio Resource Management of LTE-A Femtocells
}

\author{
Ayesha Haider Ali and Muhammad Mohsin Nazir \\ Department of Computer Science, Lahore College for Women University, Jail Road, Lahore 54000, Pakistan \\ Correspondence should be addressed to Ayesha Haider Ali; ayesha.iqbal@gmail.com
}

Received 8 March 2016; Accepted 16 June 2016

Academic Editor: Pedro M. Ruiz

Copyright ( 2016 A. Haider Ali and M. M. Nazir. This is an open access article distributed under the Creative Commons Attribution License, which permits unrestricted use, distribution, and reproduction in any medium, provided the original work is properly cited.

\begin{abstract}
The future wireless networks support multimedia applications and require ensuring quality of the services they provide. With increasing number of users, the radio resource is becoming scarce. Therefore, how should the demands for higher data rates with limited resources be met for Long Term Evolution-Advanced (LTE-A) is turning out to be a vital issue. In this research paper we have proposed an innovative approach for Radio Resource Management (RRM) that makes use of the evolutionary multiobjective optimization (MOO) technique for Quality of Service (QoS) facilitation and embeds it with the modern techniques for RRM. We have proposed a novel Multiobjective Optimizer (MOZ) that selects an optimal solution out of a Pareto optimal (PO) set in accordance with the users QoS requirements. We then elaborate the scheduling process and prove through performance evaluation that use of MOO can provide potential solutions for solving the problems for resource allocation in the advancement of LTEA networks. Simulations are carried out using LTE-Sim simulator, and the results reveal that MOZ outperforms the reference algorithm in terms of throughput guarantees, delay bounds, and reduced packet loss. Additionally, it is capable of achieving higher throughput and lower delay by giving equal transmission opportunity to all users and achieves $100 \%$ accuracy in terms of selecting optimal solution.
\end{abstract}

\section{Introduction}

LTE-Advanced (LTE-A) is an emerging wireless access network technology that has been considered the 4thGeneration (4G) wireless system to offer higher bandwidth for Internet access. It offers higher data rates up to $1 \mathrm{Gbps}$ [1]. Radio Resource Management (RRM) in LTE-A system takes into account existing spectrum both in time and in frequency domains. Fulfilling the Quality of Service (QoS) requirements is more challenging in wireless networks due to

(i) limited radio resources/spectrum,

(ii) channel conditions,

(iii) existence of multiple users with diverse QoS requirements.

The foremost goal of LTE-A systems is to improve service provisioning and reduce the cost of user and operators. This can be fulfilled by enhancing the quality of the system with reference to data rates, system capacity, coverage, throughput, and reduced latency. As certain traffic classes/flows have stringent QoS requirements, for example, video and VOIP, thus satisfying their requirements is essential to maintain network operations smoothly. As the radio resource is scarce, there is a need to have mechanisms to distribute this scarce and valuable resource efficiently to ensure QoS of individual users for development of services for next generation telecom networks. Thus, our main objective in this research is to deal with QoS in terms of throughput and delay. We aim to maximize throughput and minimize delay and these two objectives will form our objective function. Figure 1 lists the different applications and their QoS requirements in relation to delay and throughput.

In LTE-A, Physical Resource Block (PRB or simply RB) is the smallest user assignment resource unit for resource scheduling. In this allocation phase, the bandwidth is divided into portions called Resource Chunks (RCs) [2]. Variable numbers of RCs are allocated to different User Equipment 


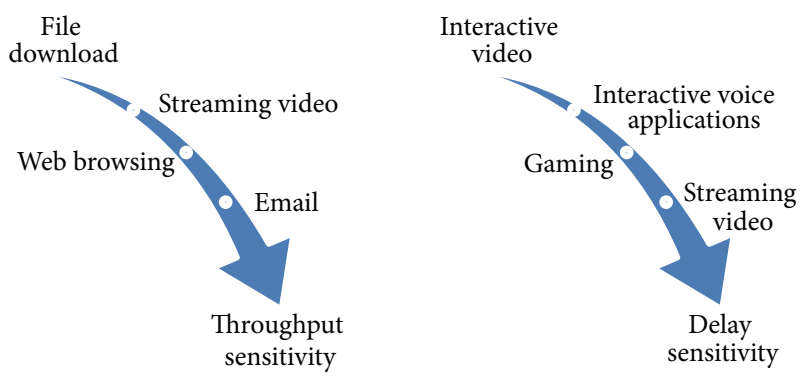

FIGURE 1: Varying QoS requirement classification.

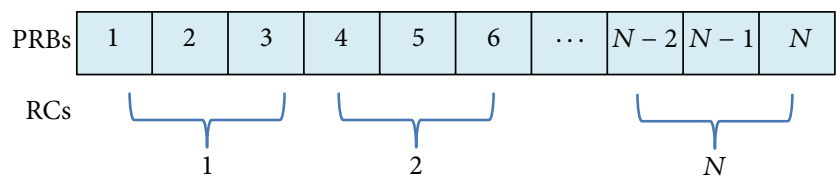

Figure 2: A total of M RCs dividing the total bandwidth into $N$ PRBs.

(UE) for various applications. Figure 2 depicts this concept. To guarantee QoS we need to select RB that will fulfill both our objectives of throughput and delay guarantees.

RRM is a set of techniques that are utilized to optimize the usage of spectrum and equipment. The motivation behind RRM functionality is to guarantee the provision of network services and QoS and optimize the system usage. The realization of a wireless system is dependent on the bandwidth allocation and the QoS gains of the transmission medium [3]. Now that data rate provisioning is heading towards the next generation, designing techniques for efficient radio resource allocation is becoming more challenging. These challenges result due to the fact that the packets arrive randomly and there is a lack of buffer space, plus the QoS needs are erratic, and thus resource management and allocation techniques need to be developed.

Our work takes advantage of the multiobjective optimization (MOO) techniques that make use of a decision maker (DM) to take radio resource allocation decisions. The sole purpose is to provide RRM technique that will ensure QoS provisioning to all types of traffic specifically for increased throughput and reduced delay. There exist evolutionary algorithms that have various schemes and techniques for managing resources [4]. In MOO, the objective vectors are viewed as optimal if none of its elements can be further improved without deteriorating at least one of the other elements [5].

Most real world problems have more than one objective function and when we have multiple objectives to attain we need efficient solutions to solve the problem. Also the relationship between objectives is generally rather perplexing and cannot be measured using the same standard thus making it hard to cumulate them into a single objective. In that case, we do not have a solitary optimal solution but rather a collection of substitutes with varying trade-offs, which are called Pareto optimal (PO) sets. Usually one of these PO solutions is selected at the end. So in multiobjective optimization we have two tasks: first finding a PO set and second a decision-making process/module that will select a distinct solution from this set also referred to as DM. Thus, when we have diverse goals with conflicting objectives they cannot be summed up into one objective function and this encourages the use of various MOO techniques.

Despite the fact that LTE deployment scenarios deliver high data rates they also showcase new challenges for interference management and RRM. To meet these challenges policies are intended for the standard cellular networks. Also the femtocells are ad hoc in nature and this fact tends to bind the scope of the algorithms under consideration. Thus, proficient RRM procedures are critical to restrain the impact of interference on LTE femtocells performance [6].

A framework for Time Domain (TD) scheduling with congestion control and QoS provisioning is also introduced. It optimizes the QoS provisioning process for domains of both time and frequency by taking into account channel condition, the status of the queues, and the user's QoS requirements. This framework enhances the process of resources utilization by considering the QoS requirements of various service classes. The results indicate better QoS of Real Time (RT) traffic and fair resource sharing of available resources for the Non-Real Time (NRT) traffic [7].

The idea of cross layer resource allocation for Orthogonal Frequency Domain Multiplexing (OFDM) scheme is considered significant as it takes into account the channel condition, the arbitrary nature of traffic, QoS needs, and fair distribution of resources between the users. Time slots, frequencies, and carriers are assigned dynamically to make this process effective [8]. A number of studies exist in literature that focus on the RRM of LTE-A considering the QoS requirements of the user. Some make use of self-optimization, some tend to follow the resource scheduling approaches, others focus on spectrum splitting using the time and frequency domains, and some have designed solution for RRM by using genetic algorithms [9-15].

The algorithms that manipulate multiuser environment are the MAX C/I and Proportional Fairness (PF) algorithms. In MAX C/I algorithm RB is allocated to a user who tends to achieve the highest data rates for the current time slot and tries to maximize overall system throughput. The PF algorithm also considers fairness and resources are allocated on the basis of priority and allocation schemes [16].

However these algorithms target to improve utilization of resources considering the channel conditions and interference mitigation only and the delay and throughput requirements are not taken into much consideration. A number of techniques have also been proposed that aim to efficiently use the radio resources to provide guaranteed QoS for various types of traffic [17-19]. A Delay Prioritized Scheduling (DPS) algorithm [20] has been developed that manages the distribution of RBs by choosing those RBs that best fulfill the delay threshold condition. With increasing users the delay also tends to increase due to more video requests that are throughput sensitive. It is a packet scheduling algorithm that selects RBs according to their SNR levels and then admits and assigns the flow to the best possible RBs. This is a twoway process which first selects a user according to a delay 
threshold and then assigns RBs. It focuses mainly on delay minimization.

In the perspective of multiobjective optimization, most work done is towards discovering a collection of nearPareto optimal solutions algorithmically. In multiobjective optimization, communication with the DM can be done during the phase of optimization or during the final decisionmaking part. In many studies a human decision maker is involved after a variety of solutions have been sought out [2123].

The process of multiobjective optimization is incomplete without a decision-making activity that will take the final decision based on various alternatives available. In this context, many interactive multiobjective optimization techniques are available under the title Multicriteria DecisionMaking (MCDM) [24-26]. All the techniques are different from each other, but all incorporate DM to provide information to help in taking the final decision. The evolutionary algorithms (EA) suggest the use of natural evolution theory for optimization like the survival of the fittest theory of Darwin. They work with a set of solutions and can also be used to find a partial PO set.

MOO has been researched for many years and is focused on the theoretical aspects [27]. A lot of approaches have been formulated with mathematical programming theory, for example, nonlinear programming to solve the multiobjective optimization problems [28]. Varying interactive approaches have also been used in which the information is given to the DM and the DM specifies its preferences. This procedure depends on the type of problem and its mathematical properties and scalar function [29].

A power-delay minimization scheme is also introduced that makes use of linear programming [30]. In this study, multiobjective problem is transformed into a solitary objective by using weighted sum technique that aims to reduce both the delay and transmit power. Simulated annealing and greedy heuristic algorithms are also part of this work. Its target is not specifically LTE networks but mainly the IEEE 802.11 Wireless LANs (WLANs) and Green Wireless Access Networks (GWANs)

Table 1 depicts the classification of various techniques used for RRM and for finding an optimal solution with multiple objectives. Based on the literature survey, we can see that there exist various techniques for QoS provision in LTE-A using different methodologies. Also the multiobjective optimization offers a variety of solutions for solving various linear and nonlinear problems. Still, there is a lack of these multiobjective optimization technique applications for RRM of LTE-A targeted towards ensuring QoS. As a result, we will propose a QoS aware Multiobjective Optimizer (MOZ) for the RRM of LTE-A which will take into account the provision of maximum throughput and minimum delay.

\section{Materials and Methods}

2.1. Problem Formulation. To measure the performance of LTE-A network we have a set of criteria such as throughput, end-to-end transmission delay, energy efficiency, and
TABLE 1: Classification of literature on RRM and optimization.

\begin{tabular}{lcccccc}
\hline Approach & [7] & [16] & [20] & [21-23] & [30] & Proposed approach \\
\hline Scheduling & $\sqrt{ }$ & $\sqrt{ }$ & $\sqrt{ }$ & $\sqrt{ }$ & $\sqrt{ }$ & $\sqrt{ }$ \\
User association & $\sqrt{ }$ & $\sqrt{ }$ & $\sqrt{ }$ & - & - & $\sqrt{ }$ \\
QoS aware & $\sqrt{ }$ & $\sqrt{ }$ & $\sqrt{ }$ & - & $\sqrt{ }$ & $\sqrt{ }$ \\
Energy efficiency & - & $\sqrt{ }$ & - & - & $\sqrt{ }$ & - \\
RB allocation & - & - & $\sqrt{ }$ & - & $\sqrt{ }$ & $\sqrt{ }$ \\
LTE & $\sqrt{ }$ & - & - & - & $\sqrt{ }$ & - \\
LTE-A & - & $\sqrt{ }$ & $\sqrt{ }$ & - & - & $\sqrt{ }$ \\
MOO & - & - & - & $\sqrt{ }$ & $\sqrt{ }$ & $\sqrt{ }$ \\
DM & - & - & - & $\sqrt{ }$ & - & $\sqrt{ }$ \\
\hline
\end{tabular}

transmission strength. The purpose of the work presented here is to determine the trade-offs that arise while choosing performance metrics for assigning resources to the User Equipment (UE). The multiobjective Pareto optimization consists of three steps:

(1) Multiobjective problem definition.

(2) Optimization (finding Pareto optimal solutions).

(3) Decision-making (role of DM).

In the following we will elaborate the working of our QoS aware optimizer according to the above-mentioned three steps and it will define the framework for our proposed optimizer.

Step 1 (problem definition). To solve our multiobjective optimization problem we will involve DM to find the best (optimal) solution. By optimal solution we are referring to Pareto optimal solution that the DM considers as the best option. In our case, we have two objectives: the first one is maximum throughput and the second one is minimum delay. We will manage the resources in such a way that we are able to select $\mathrm{RB}$ that transmits the data according to the abovementioned objectives.

Pareto Optimization Problem. We will solve the multiobjective optimization problem that will take the form

$$
\begin{aligned}
\text { Maximize/Minimize } & \left\{f_{1}(y), f_{2}(y), \ldots, f_{i}(y)\right\} \\
\text { Subject to } & y \in S
\end{aligned}
$$

where $i$ is a set of objectives that will be minimized or maximized according to its definition. $S$ is a set of network constraints. Here, $y$ is the decision vector and $y^{\prime}$ is the decision variable. We will call $y^{\prime} \in S$ Pareto optimal only if there is no other $y^{\prime} \in S$ such that

$$
f_{k}(y) \leq f_{k}\left(y^{\prime}\right) \quad(\text { where } k=1, \ldots, i) .
$$

This means that $y^{\prime}$ is Pareto optimal only if there is no other possible vector (or solution) $y$ that will deteriorate some criterion without leading towards an increase in some other criterion. 
Step 2 (Pareto front). In our case, we have the following objective functions and the first one aims at maximizing network utility through increased throughput $\left(T_{i}\right)$ :

$$
\begin{array}{ll}
\text { Maximize } & \sum_{i=1}^{n}\left(T_{i}\right) \\
\text { Subject to } & \sum_{i=1}^{n} T_{i}<C_{i, k}(t) \quad \text { where } T_{i}>0 \forall i \in N \\
& (N=\text { total UE instances }) .
\end{array}
$$

Considering channel $k$ and user $i$ we define the channel capacity as

$$
C_{i, k}=\mathrm{RB} \log _{2}\left(1+\frac{\rho}{\sigma^{2}} \cdot P\right),
$$

where $\mathrm{RB}$ is $\mathrm{RB}$ Bandwidth which is calculated as (total bandwidth/number of RCs), $P$ is Transmission Power, $\rho$ is SNR space determined by Bit Error Rate (BER), and $\sigma^{2}$ is noise power density.

$T_{i}$ is the average throughput calculated as

$$
T_{i}=\operatorname{TUE}_{i}[t] \frac{1}{\tau}+T_{i}(t-1)\left(1-\frac{1}{\tau}\right) \quad \forall i \in N,
$$

where $\mathrm{TUE}_{i}[t]$ is throughput of $\mathrm{UE}_{i}$ in time instance $t, \tau$ is time constraint of the smoothing filter, and $N$ is total UE.

$\mathrm{TUE}_{i}[t]$ is calculated with the help of the following equation:

$$
\operatorname{TUE}_{i}[t]=\mathrm{RB} \log _{2} 1+\mathrm{SNR},
$$

where SNR $=T_{p} \times S_{\mathrm{UE}} /($ Noise + Interference $)\left(T_{p}\right.$ is Transmission Power; $S_{\mathrm{UE}}$ is signal gain of UE).

Our next objective is defined as follows:

$$
\begin{aligned}
\text { Minimize } & \sum_{i=1}^{N}\left(D_{i}(t)\right) \\
\text { Subject to } & D_{i}(t)<\mathrm{DB}_{i},
\end{aligned}
$$

where $\mathrm{DB}_{i}$ is the delay budget or the upper bound which is equivalent to $20 \mathrm{~ms}$ in OFDMA networks $[19,31]$. The delay experienced by the $\mathrm{UE}_{i}$ should be less than this upper bound. Here, $N$ represents total active $\mathrm{UE}$ and $D_{i}(t)$ is the HOL (Head of Line) delay for $\mathrm{UE}_{i}$ at time $t$ calculated as

$$
D_{i}(t)=\frac{W_{i}}{\mathrm{DT}_{i}},
$$

where $W_{i}$ is the waiting time for $\mathrm{UE}_{i}$ and $\mathrm{DT}_{i}$ is the normalized HOL delay obtained by dividing each user's waiting time by $\mathrm{DB}_{i}$.

This process will generate a set of Pareto optimal solutions called the Pareto front. As a result there is no single PO solution so the question is which solution to select from a set of PO solutions. The answer lies in Step 3 that takes advantage of DM.
Step 3 (the optimal solution). In this step the DM will select the best solution, which is RB that will be allocated for transmission. Depending on the type of incoming traffic the DM will decide which strategy to follow according to the following criteria:

(i) If the traffic is delay sensitive, preference will be given to minimizing the delay whereas throughput can be compromised to some extent.

(ii) If the incoming packet is throughput sensitive, it will emphasize maximum throughout whereas delay may be compromised.

This scheme will thus allow the delay sensitive applications to sacrifice throughput for lower delays without any effect on the throughput sensitive applications.

So we have $k$ and $i$ objective functions (represented by $g$ ) and a total of $m$ and $f$ constraints (represented by $h$ ). We assume that the constraints plus the objective function are the functions of the decision vectors. So, the DM's goal is as follows:

$$
\begin{array}{ll}
\text { Maximize } & g(x)=\left\{g_{1}(y), g_{2}(y), \ldots, g_{i}(y)\right\} \\
\text { Minimize } & g(y)=\left\{g_{1}(z), g_{2}(z), \ldots, g_{k}(z)\right\} \\
\text { Subject to } & h(x)=\left\{h_{1}(y), h_{2}(y), \ldots, h_{m}(y)\right\} \\
& h(y)=\left\{h_{1}(z), h_{2}(z), \ldots, h_{f}(z)\right\} \\
& x=\left\{x_{1}, x_{2}, \ldots, x_{i}\right\} \\
& z=\left\{z_{1}, z_{2}, \ldots, z_{k}\right\}
\end{array}
$$

where $x$ is the decision vector for first objective function and $z$ is the decision vector for second objective function. $O^{*} \epsilon$ $x$ or $z$ is the feasible decision or solution to our optimization problem. $O^{*}$ is Pareto optimal as we have no other better solution. So here the DM will make use of a weighted MinMax Approach which is adopted from Game Theory $[32,33]$. In this scheme we will compare the relative deviation from a separately attainable minima or maxima. In our case it is the deviation from maximum throughput and delay of the system. We will denote this by $D_{\max } i$ (delay variation) and $T_{\min } i$ (throughput variation). We will calculate the relative deviations using

$$
T_{\min } i=\mathrm{TA}_{s}-T_{i}
$$

(For throughput sensitive applications).

$\mathrm{TA}_{s}$ is the maximum attainable throughput of the LTE system calculated as follows.

(i) We will first calculate the total RBs assuming the bandwidth of channel is $20 \mathrm{MHz}$ by using the following:

$$
\text { carriers } \times \text { OFDM symbols } \times \text { Slots } \times \text { RBs, }
$$

where carriers are 12. OFDM symbols are 7. RBs are 100. Slots are 2 . The final value is $16800 \mathrm{RBs}$ per frame. 
(ii) Second, we consider modulation of 64 QAM and a single modulation symbol carries 6 bits. The total bits will thus be

16800

$\times 6$ bits per symbol of modulation equal to $100.8 \mathrm{Mb}$.

(iii) Thirdly, considering the 4-by-4 MIMO, we get

$$
4 \times 100.8 \mathrm{Mb}=403 \mathrm{Mb} \text {. }
$$

This is the peak data rate.

(iv) Finally, we calculate the overhead which will be about $25 \%$. So, we have

$$
403 \mathrm{Mb} \times 0.75=302 \mathrm{Mb} .
$$

So, we can calculate $\mathrm{TA}_{s}$ and $\mathrm{RC}$ and minimum $T_{\min } i$ is selected by the DM for transmission. That is resource chunk $\mathrm{RC}_{i}$ as in the following:

$$
O^{*}=\mathrm{RC}_{i}=\min T_{\min } i, \quad \forall i \in \mathrm{UE} .
$$

Now, let us talk about the delay sensitive applications where we will find the feasible solution $\mathrm{O}^{*}$ as follows:

$$
D_{\max } i=\mathrm{DB}_{i}-D_{i}(t)
$$

(For delay sensitive applications)

$$
\mathrm{O}^{*}=\mathrm{RC}_{i}=\max D_{\max } i, \quad \forall i \in \mathrm{UE} \text {. }
$$

So $\mathrm{O}^{*}$ is chosen for transmission based on the following decision vectors:

$$
O^{*}= \begin{cases}\arg \min \left\{T_{\min } i\right\} & \forall \mathrm{UE}_{i} \\ \arg \max \left\{D_{\max } i\right\} & \forall \mathrm{UE}_{i} .\end{cases}
$$

The DM will take its decision based on the following set of constraints:

$$
\begin{aligned}
& \sum_{i=1}^{n} T_{i}<C_{i, k}(t) \quad \text { where } T_{i}>0 \forall i \in N \\
& D_{i}(t)<\mathrm{DB}_{i} \quad \forall i \in N \\
& \sum_{i=1}^{N} \mathrm{RB}_{i} \leq \text { Total Badndwidth } \\
& \sum_{j \in J} \theta_{i, j} \geq 1 \quad \forall i \in N, \forall J \in \text { Total BS. }
\end{aligned}
$$

Equation (18) refers to channel capacity constraint, and (19) refers to delay bound constraint. Equation (20) is RB bandwidth constraint. Equation (21) ensures that at least $1 \mathrm{BS}$ covers the active UE.

2.2. The Decision-Making Process. After the optimized solution is sought out by the DM, the traffic will then be scheduled in output queues discussed in detail in the next subsection.
Figure 3 depicts the detail of decision-making process inside the DM. Its details are explained as follows.

(1) Traffic Type Determination. The DM will take as input the Pareto front and will first determine the type of traffic which in our case is either throughput or delay sensitive.

(2) Optimization Process. After determining the traffic type the following actions are taken:

(i) For throughput sensitive applications it will calculate the value of $T_{\min } i$ based on the value of $T_{i}$, and then it will select the optimal solution $\mathrm{O}^{*}=\mathrm{RC}_{i}=$ $\min T_{\min } i$.

(ii) For delay sensitive applications it will calculate the value of $D_{\max } i$ based on the value of $D_{i}(t)$, and then it will select the optimal solution $O^{*}=\mathrm{RC}_{i}=$ $\max D_{\max } i$.

(3) RC Allocation Matrix Update. After that the RC assignment matrix will be updated. The matrix $m_{k, n}$ is represented as

$$
m_{k, n}= \begin{cases}0, & \text { if } \mathrm{RC}_{n} \text { is unassigned } \\ 1, & \text { if } \mathrm{RC}_{n} \text { is assigned to service group } k,\end{cases}
$$

where $k$ is type of traffic and $n$ is RC number [34].

(4) RC Availability Check. If there are RCs available/left for assignment, then the process starts again and moves to traffic type determination; otherwise the process ends.

In the following we explain in detail the construction of Pareto front.

2.3. Constructing Pareto Front. This step describes in detail the process of constructing a representative Pareto front as follows.

Step 1. Determine the traffic type of the incoming packet. Assume $D_{1}, \ldots, D_{n}$ are the alternative solutions in terms of $i$ and $j$, where $i$ represents throughput sensitive flows and $j$ represents delay sensitive flows.

Step 2. Find the largest $i$ such that $D_{i}>D_{n}$ (where $D_{n}$ represents all other UE), and add $D_{i}$ to Pareto front.

Step 3. Find the smallest $j$ such that $D_{j}<D_{n}$ (where $D_{n}$ represents all other $\mathrm{UE}$ ), and add $D_{j}$ to Pareto front.

Step 4. Repeat Steps 2 and 3 until no such $i$ and $j$ exist and we have the Pareto front of the form $\left(D_{i}, \ldots, D_{n}, D_{j}, \ldots, D_{n}\right)$.

Note that $D_{i}$ is sorted in order of decreasing throughput and $D_{j}$ is sorted in order of increasing delay. The process generates a set of candidate solutions that will undergo the optimization phase for selecting a singled optimal solution. The selection of the optimal solution is based on the efficiency of the DM which not only considers the resource requirements of the users but also sets priorities. We now present an algorithm that will make use of the defined framework and considers the requirements of each step. 


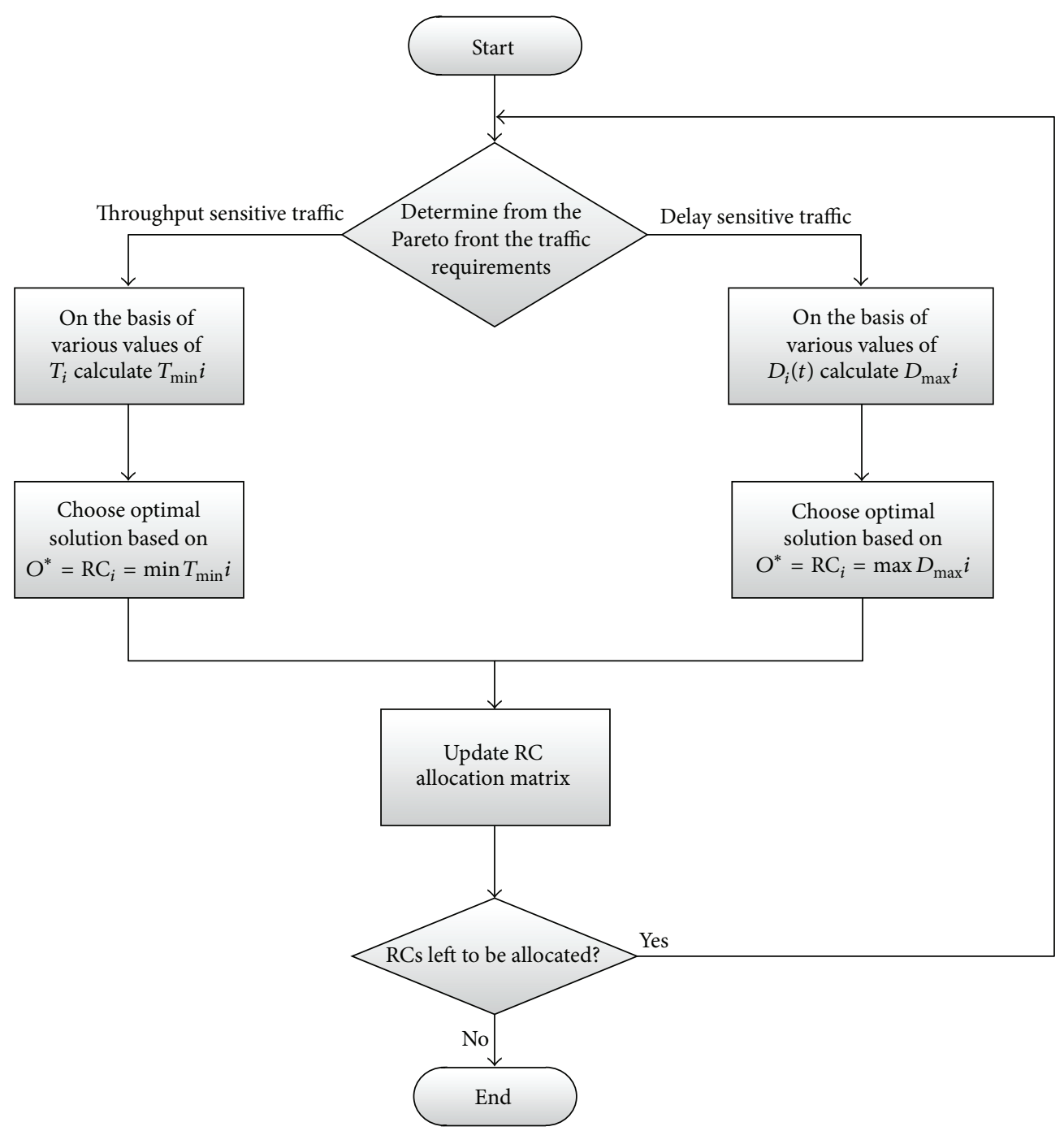

FIGURE 3: The decision-making process inside the DM for RC selection based on the objectives of maximum throughput and minimum delay.

2.4. The Multiobjective Optimizer Algorithm. The detailed working of proposed MOZ is outlined in Figure 4. The MOZ consists of three main steps as follows.

Step 1. The traffic classifier will take as input traffic from various UE instances and will divide them into 2 queues; one consists of packets that have stringent throughput requirement and the other queue consists of packets from delay sensitive applications.

Step 2. The information from Step 1 is then passed onto the optimizer that will find out the Pareto front and will send its decision set to the decision maker.

Step 3. The DM will make the final decision based on the information it has and according to its decision-making capabilities.

Step 4. Finally $O^{*}$ is sent out to the queue for scheduling and the process then diverts to Step 2 to get the next $O^{*}$ later to be scheduled.
Step 5. The process is completed when either no RC is left or there are no more incoming packets.

Steps 2 and 3 are interrelated as the DM is way dependent on the input it gets from the Pareto front, so constructing a legitimate Pareto front is itself a very important task for MOZ. The decision-making process inside the DM is characterized by a number of factors already explained and is one of the major components of our proposed strategy.

Compared to most algorithms and frameworks designed to ensure QoS and to manage RRM, the distinct differences of our proposed approach are as follows: (1) it makes use of multiobjective optimization that deals with conflicting objectives simultaneously instead of converting them into a single objective. (2) It makes use of a decision maker that has the decision-making capability and works based on the information it has and various algorithms used. (3) Multiple UE instances are served based on the type of incoming traffic/flow instead of allocating RBs to just one flow. 


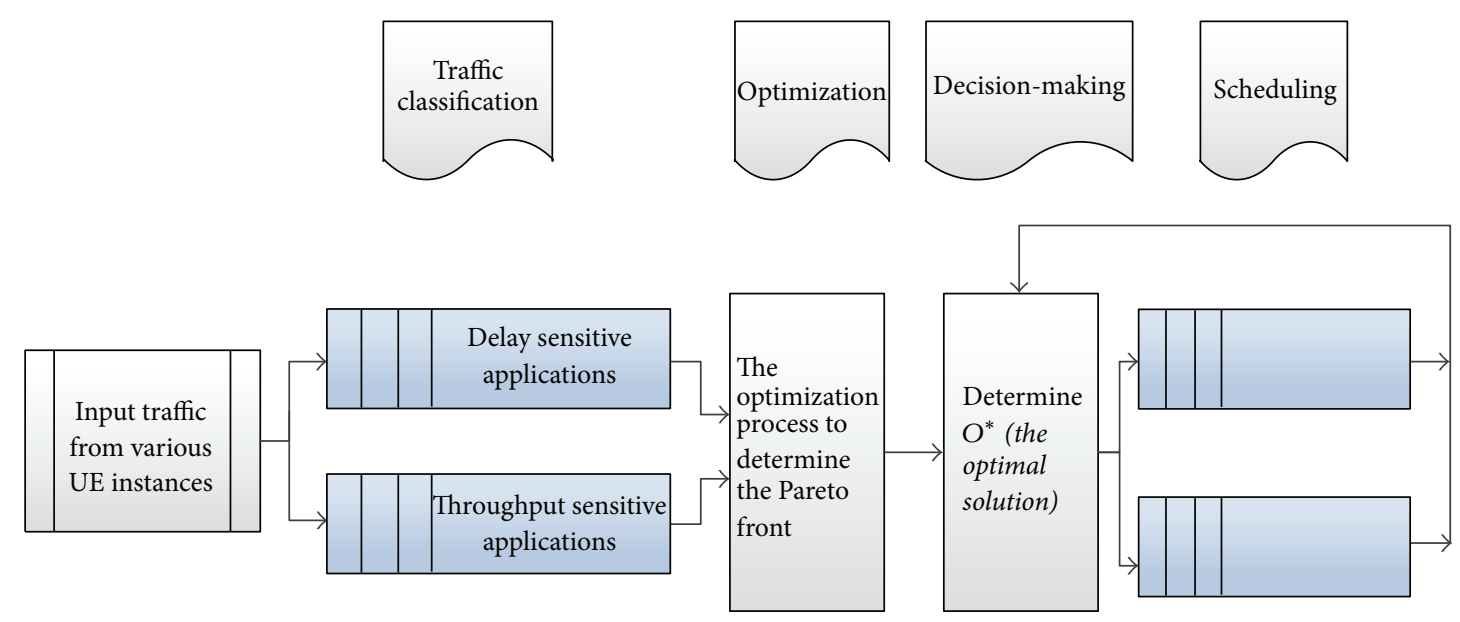

FIgURE 4: The proposed MOZ.

The goal of using MOZ is to always get an optimal solution which is chosen from a set of solutions in the form of Pareto front.

2.5. Queuing and Scheduling. Now that we have the Pareto optimal solution in hand, the packets are now ready to be scheduled in their respective queues for transmission as follows.

Step 1. In this step we will define the priority metric for delay sensitive flows. The priority of $\mathrm{UE}_{i}$ at time $t, P_{d i}(t)$, is

$$
P_{d i}(t)=D_{\max } i * G_{i, j},
$$

where $G_{i, j}$ is channel gain of $\mathrm{UE}_{i}$ on $\mathrm{RC}_{j}$ calculated as

$$
\begin{aligned}
G_{i, j}= & 10^{\text {Path loss } / 10} * 10^{\text {shadow fading } / 10} \\
& * 10^{\text {multipath fading } / 10}
\end{aligned}
$$

Step 2. We will then form a delay queue for a given $\mathrm{UE}_{i}$ that will ensure delay bounds as follows:

$$
D_{i}(t+1)=\left[D_{i}(t)-\rho R_{i}(t)\right]+\sum_{i=1}^{N} Q_{i}(t),
$$

where $Q_{i}(t)$ is the mean queue length at time interval $t$ and $N$ is total UE scheduled and $\rho R_{i}(t)$ is the service rate. In each time interval the UE with highest priority occupies front position in the queue.

Step 3. For the priority metric of throughput sensitive flows we will use the following:

$$
P_{t i}(t)=\sigma_{i} * T_{\min }(i),
$$

where $\sigma_{i}$ is the Head of Line (HOL) blocking and $P_{t i}(t)$ is the priority metric for throughput sensitive applications. In each time interval the UE instances are scheduled according to the ascending order of their priority values.
It follows the following constraints:

$Q_{i, k}$ s.t. $\forall i \in N, k \in\{$ delay sensitive traffic only $\}$

$Q_{i, j}$ s.t. $\forall i \in N$ and $j$

$\epsilon\{$ throughput sensitive traffic only $\}$

$i \neq j$, Queue Length

$$
=\operatorname{Length}\left(Q_{i k}+Q_{i j}\right) \text {. }
$$

Equation (27) ensures that the queue $Q_{i}$ takes as input delay sensitive traffic only, (28) ensures that the queue $Q_{i, j}$ takes in throughput sensitive traffic only, and (29) states that queue length is the total of both the queues.

Thus, the defined framework and the optimizer will work together to form our proposed model. The whole process has four main goals:

(1) Determining the target objectives.

(2) Based on the objectives, forming a system model that will take into account the objective functions to be minimized or maximized.

(3) Constructing a representative solution pool to aid the decision maker.

(4) Introducing a decision-making entity that will not only ease the task of scheduling but also find an optimal solution to satisfy the defined objectives.

In the following section we will define the simulation model and will discuss the results.

\section{Simulation and Results}

3.1. Simulation Model. Designing the model for LTE-A performance evaluation is generally more complex compared to a $3 \mathrm{G}$ system. An interface is used between various evolvedNodeBs (eNBs, i.e., the BS) in the LTE system to ease the traffic load that occurs due to handovers. In this 
TABLE 2: Simulation parameters.

\begin{tabular}{lc}
\hline Parameters & Values \\
\hline Carrier frequency & $2 \mathrm{GHz}$ \\
Bandwidth & $10 \mathrm{MHz}$ \\
Number of subcarriers & 300 \\
Number of RBs & 45 \\
TTI & $1 \mathrm{~ms}$ \\
Path loss model & Free space propagation model \\
Transmit power & $30 \mathrm{~mW}$ \\
Fading model & Rayleigh \\
Packet model & Poisson model \\
Multiplexing & MIMO \\
Number of UE instances & 20 \\
\hline
\end{tabular}

section we will design the simulation model for testing and will evaluate our system for examining delay, throughput, Packet Loss Ratio (PLR), the effect of SNR, and cell spectral efficiency.

We are considering a cell with the capacity of $10 \mathrm{MHz}$ and it consists of $45 \mathrm{RBs}$ and the carrier frequency is $2 \mathrm{GHz}$. All the available RBs are controlled by the eNodeB. The RBs are divided among the UE instances in the form of RCs. The LTEA parameters used for simulation are described in Table 2.

We then calculate the number of bits in each RB (i.e., its data rate). It can be computed as follows:

$$
b_{i, \mathrm{RC}}=\mathrm{NS}_{\mathrm{tti}} * \mathrm{NS}_{s} * S_{\mathrm{rb}} \text {, }
$$

where $b_{i, \mathrm{RC}}$ is achievable data rate, $\mathrm{NS}_{\mathrm{tti}}$ is number of slots in each TTI, $\mathrm{NS}_{s}$ is number of symbols in each slot, and $S_{\mathrm{rb}}$ is number of subcarriers in each RB.

Each UE is assigned a queue according to the traffic type and parameters as described in the system model previously. After that we will calculate the channel gain of each $\mathrm{UE}_{i}$ on $\mathrm{RC}$, and we will now proceeed to calculate the SNR.

The SNR values are reported to ENodeB by the UE at each TTI. All the users have a different value of SNR according to the experienced fading and multipath propagation, the transmit power $(P)$, and the total RBs $(N)$ as follows:

$$
\mathrm{SNR}=\frac{P * G_{i, j}}{N(\text { Noise }+ \text { Interference })} .
$$

The incoming packets will be treated by the eNodeB according to the parameters defined in Table 2 and the system model presented. All packets have varying length and are streamed in queues according to their priorities and properties. Now, for the delay sensitive packets the threshold $\mathrm{DB}_{i}$ is set to be $20 \mathrm{~ms}$ for each UE.

Thus we can define the Average System Delay (AvD) with a total simulation time of $T$ at time instance $t$ with total number of UE instances equal to $N$ as follows:

$$
\operatorname{AvD}=\frac{1}{T} \sum_{t=1}^{T} \frac{1}{N} \sum_{i=1}^{N} D_{i}(t) .
$$

We will then define AvTH which is the average throughput with trans ${ }_{i}(t)$ being the number of packets/sec, as follows:

$$
\operatorname{AvTH}=\frac{1}{T} \sum_{i=1}^{N} \sum_{t=1}^{T} \operatorname{trans}_{i}(t) .
$$

Now for evaluating the PLR by using the size of discarded packets $\operatorname{pdisc}_{i}(t)$ and the sum of sizes of all packets psize $i_{i}(t)$, we have

$$
\operatorname{PLR}=\frac{\sum_{i=1}^{N} \sum_{t=1}^{T} \operatorname{pdisc}_{i}(t)}{\sum_{i=1}^{N} \sum_{t=1}^{T} \operatorname{psize}_{i}(t)} .
$$

The overall spectrum efficiency $\left(S_{\text {eff }}\right)$ is measured in terms of total traffic usage factor $T_{f}$ by applying the formula

$$
S_{\mathrm{eff}}=\frac{C_{\mathrm{tot}}}{T_{f}},
$$

where $T_{f}=r \times p$, where $r$ is the resource usage factor and $p$ is total traffic. $C_{\text {tot }}$ is number of available traffic channels in the system.

The queuing model is set up by considering $X_{t}$ that represents the number of UE instances at a given time interval $t \in T$ and $U_{t}$ denotes the number of new UE instances that arrive at time $t \in T$ with common probability density function $f$ on $T$, and we define

$$
X_{t+1}=\left\{\begin{array}{ll}
U_{t+1} & X_{t}=0 \\
\left(X_{t}-1\right)+U_{t+1} & X_{t}>0,
\end{array} \quad t \in T,\right.
$$

where $X=\left\{X_{0}, X_{1}, \ldots\right\}$ is a Markov Chain that has the probability matrix of the following form:

$$
\begin{aligned}
& P(0, y)=f(y), \quad y \in T \\
& P(y, z)=f(y-z+1), \\
& \quad z \in T, y \in(z-1, z, z+1, \ldots) .
\end{aligned}
$$

The chain $X$ defined here is the queuing chain of distribution $f$, and starting from $y \in T$ a UE instance is served and new UE instances arrive by the next time unit $t$ controlled by $f$. So the probability of going from state $y$ to $z \in(y-1, y, y+$ $1, \ldots)$ is $\lfloor(z-y+1)\rfloor$.

3.2. Results. We will now discuss the simulation results based on the simulation model and proposed strategy. In this paper, we have used the LTE-SIM [35] simulator available online at http://telematics.poliba.it/LTE-Sim. It is crucial to carry out performance evaluation for LTE-A systems and this requires the use of standard simulators. LTE-Sim is designed according to the LTE-A standard with main features such as multicell environment, multiuser support, eNodeB, CQI, UE, resource allocation, QoS management, and scheduling strategies.

The network topology under consideration comprises network nodes (including eNodeBs and UE), distributed among various cells. We have tested our algorithms for both 


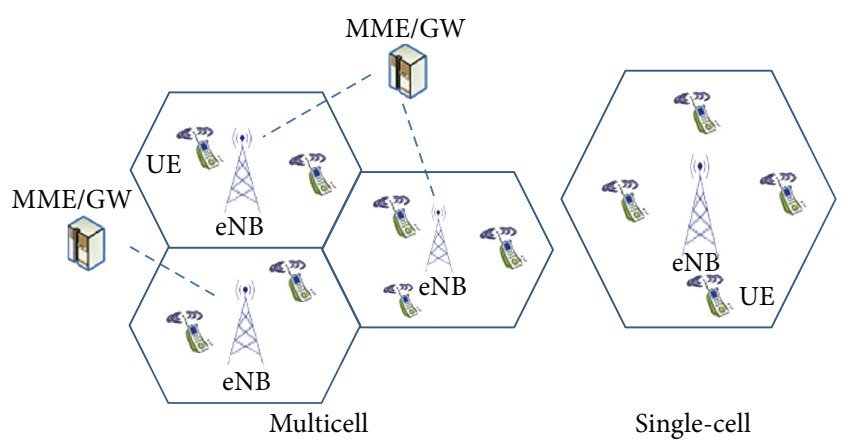

FIGURE 5: Single-cell and multicell simulation scenarios.

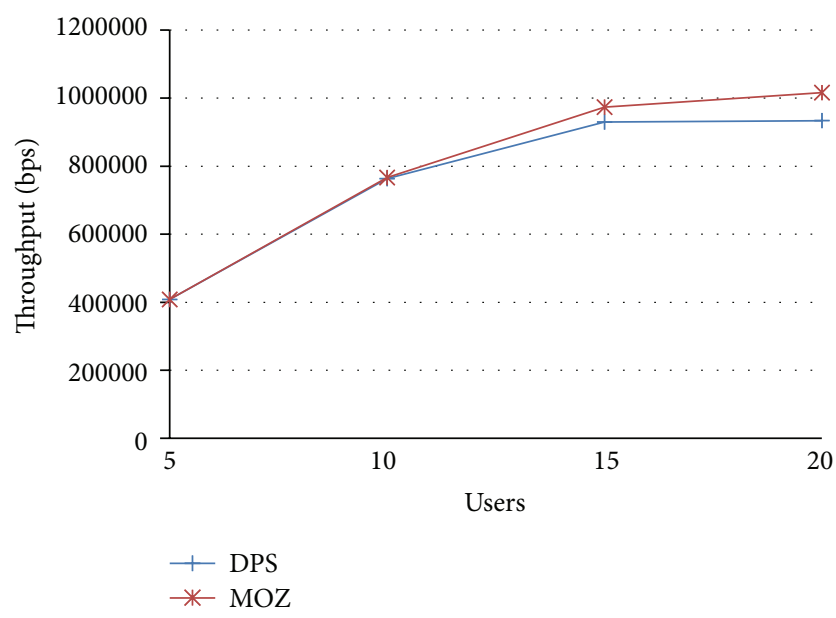

FIGURE 6: Video throughput.

the single-cell and multicell scenario as shown in Figure 5. The first scenario consists of many eNodeBs due to the fact that multiple cells are covered while in the second scenario one eNodeB handles all UE.

We will evaluate the performance of our proposed MOZ while comparing it with that of DPS [20]. For each UE, downlink VOIP and video flows are considered. All flows are active during the whole simulation. Time for each simulation is around $100 \mathrm{~s}$. The simulation is performed using a Windows machine with Cygwin (Linux-like environment), a $2.6 \mathrm{GHz}$ CPU, and 4 GB RAM.

3.2.1. Delay and Throughput. The goal of achieving desired level of QoS is very challenging due to Real Time multimedia applications, which have strict delay and throughput constraints. Figures 6 and 7 show the throughput for video and VOIP flows with increasing number of UE instances. From both the figures, it can be seen that the throughput achieved by MOZ is better than that of DPS algorithm although the difference for VOIP flow is much less. Throughput also increases with increasing number of users. For video, up to 15 users increasing pattern is observed and after 25 users throughput increases but gradually. For VOIP flows the throughput is still increasing although it is delay sensitive. The reason behind this increased throughput is that the system resources are utilized according to the requirement of users

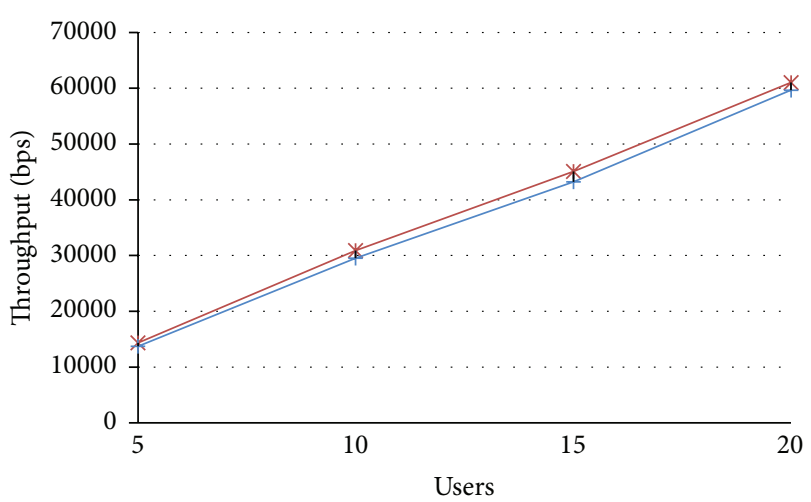

* MOZ

FIGURE 7: VOIP throughput.

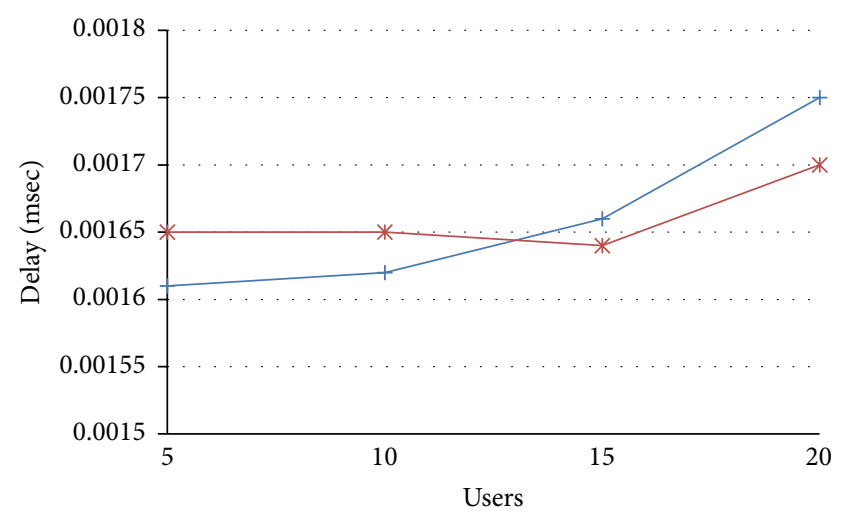

+ DPS

* MOZ

FIgURE 8: VOIP delay.

and only the selected UE instances transmit on the best RBs. Also it is worth noticing that the throughput achieved by MOZ is much closer to the theoretical maximum. In this process decisions are made based on the channel quality of each UE, which constantly measures the QoS parameters based on the algorithms and techniques proposed. After 10 users, the throughput for video using MOZ achieves performance gains in order of 1 to $15 \%$, while for VOIP it is from 1 to $10 \%$.

Figures 8 and 9 show the average delay of the MOZ and DPS algorithm with increasing UE for both the video and VOIP flows. From Figure 8, it can be seen that the delay performance of MOZ is slightly higher compared to DPS with less than 10 users and as number of UE instances increases MOZ shows better performance compared to the DPS algorithm because after 10 plus users there is more video streaming and it is not distinguishing VOIP and video flows so the delay for DPS will increase in consecutive TTIs. Figure 9 shows the performance of both MOZ and DPS which is very close because with increasing users video demand has a possibility of rising.

Also our results ensure that the delay always remains below the threshold value. The proposed MOZ achieves 


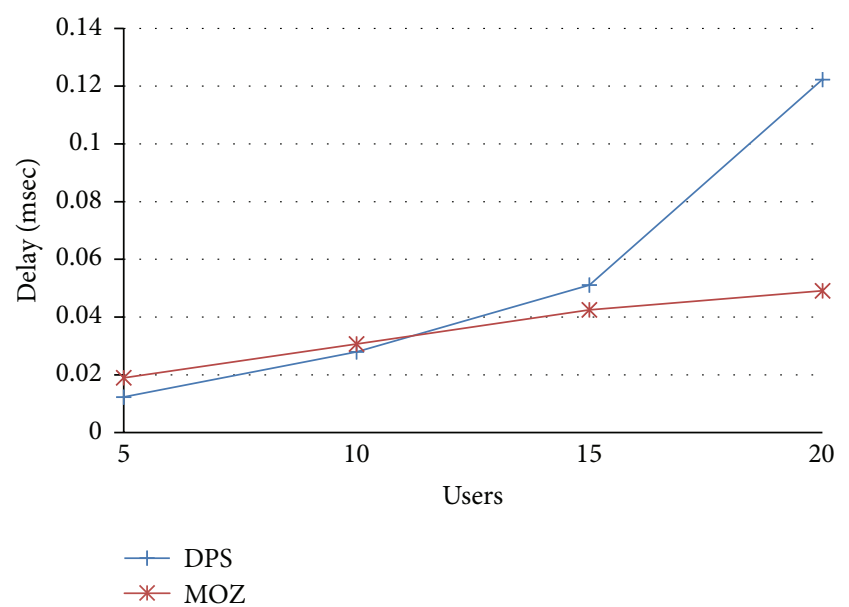

Figure 9: Video delay.

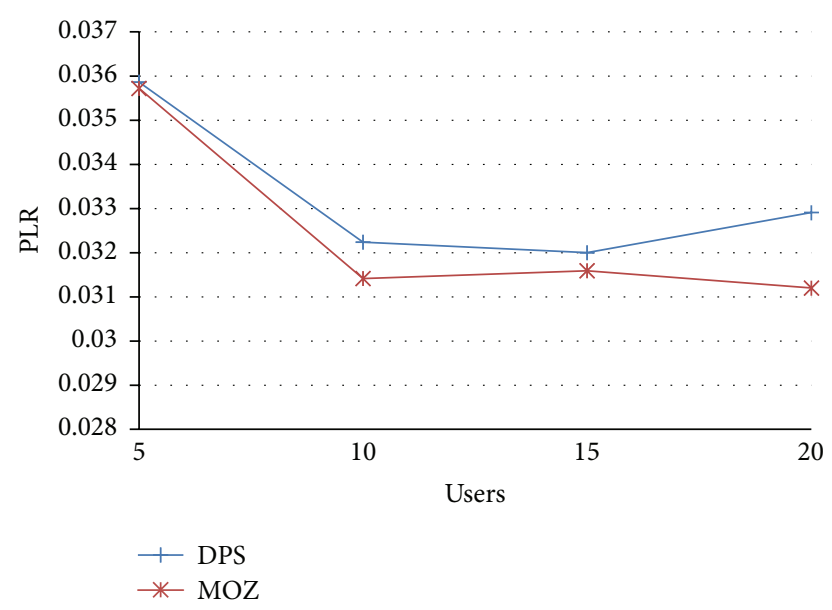

FIGURE 10: Packet loss ratio.

performance gains by reducing delay from 1 to $5 \%$ for video and 1 to $8 \%$ for VOIP after 10 users. Also it is worth noticing here that the delay achieved is way below the threshold which was set to $20 \mathrm{~ms}$.

3.2.2. PLR. Now let us compare the PLR performance of MOZ and DPS algorithm shown in Figure 10. As expected, the PLR is increasing as the number of users increases because there are more packets that will be discarded as there will not be sufficient RBs for transmitting all the packets considering that the packets are nearing their delay threshold. However, in comparison with the DPS algorithm, MOZ is capable of achieving a better PLR as it supports more users by distinguishing packets according to their scheduling and resource needs. It reduces the loss probability by exploiting the gains at physical layer. Note that the PLR counts only for physical layer losses. MOZ achieves a better PLR and packet loss is reduced from 2 to $14 \%$ as compared to the reference algorithm.

3.2.3. SNR. Figure 11 shows the SNR performance of MOZ with reference to average system throughput. With increasing

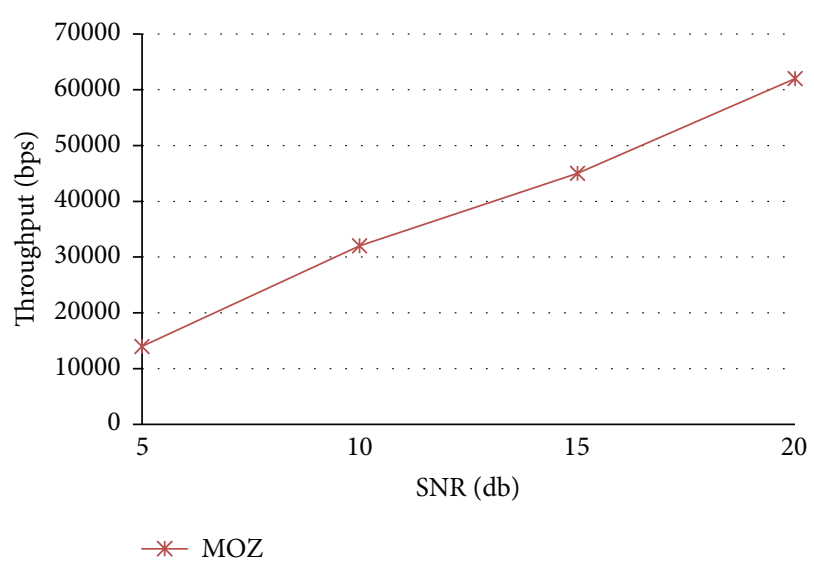

FIGURE 11: SNR versus throughput.

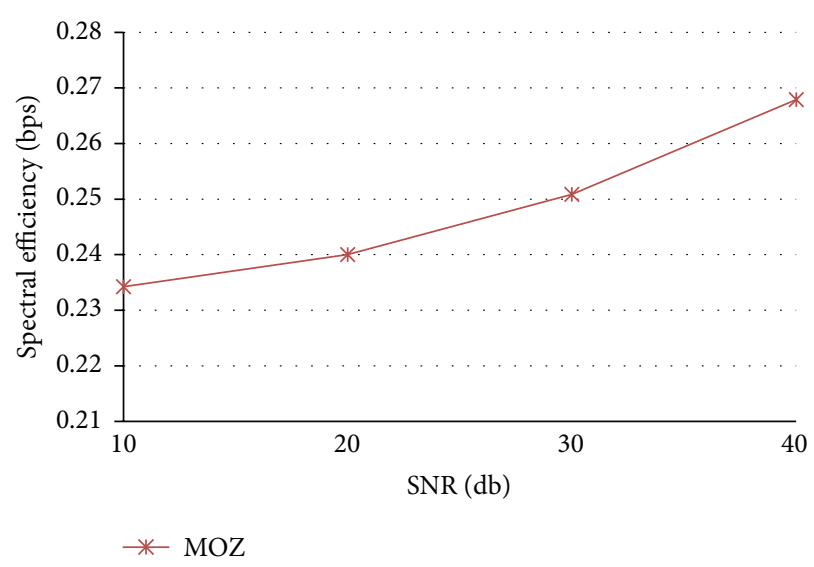

FIGURE 12: Cell spectral efficiency.

throughput it is observed that the SNR is also increasing which depicts better channel quality and less distortion and is one of the reasons we are able to achieve higher system throughput. The higher values of SNR are indicator of higher percentage of data as compared to noise and our results show that noise is around $0.04 \%$ of the actual signal which is an indicator that the video and voice quality achieved are excellent.

3.2.4. Spectral Efficiency. Then the spectral efficiency (in bits/s) is computed and is depicted in Figure 12. At each scheduling interval, the $\mathrm{RB}$ is allocated to $\mathrm{UE}$ instances according to their needs. As the number of UE instances increases MOZ still guarantees QoS constraints to more flows with a positive effect on overall system efficiency. The system supports delay and throughput guarantees simultaneously; therefore, the spectral efficiency increases with increasing users which is a good indicator of overall system gain and efficiency.

3.2.5. Energy and Memory Consumption. Figure 13 demonstrates the energy consumption in joules with respect to the number of users in the network. The energy usage of MOZ is compared to PF scheduling and DPS algorithm. As the 


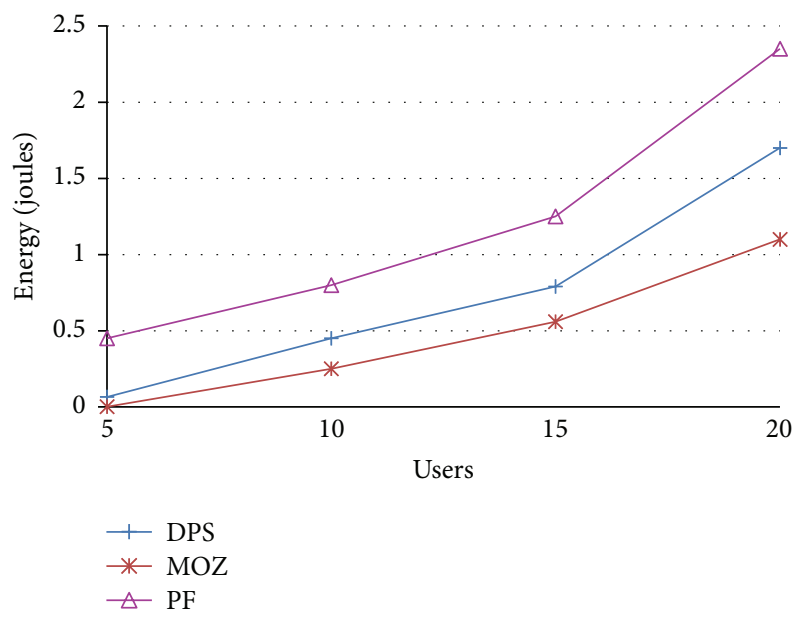

FIGURE 13: Energy consumption versus no. of nodes.

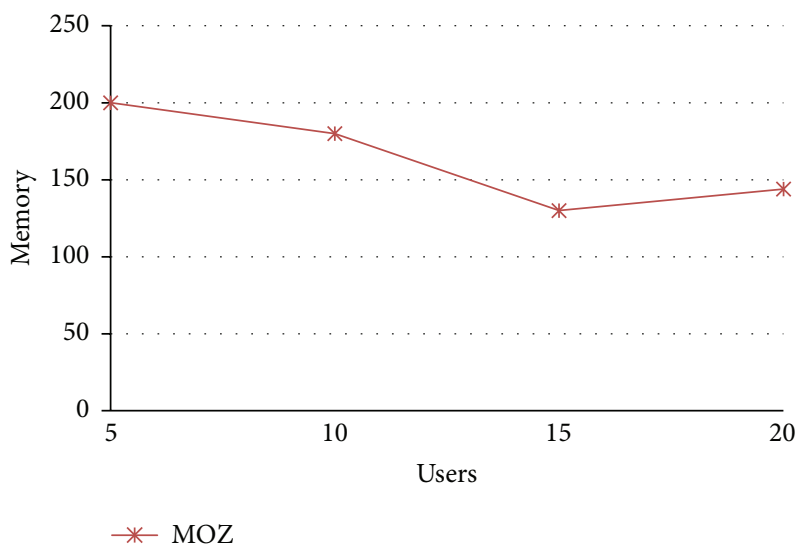

FIGURE 14: Memory consumption versus no. of nodes.

number of users increases the level of energy consumption also rises for all algorithms. As the eNodeB remains active all the time this rise is expected. But the energy consumption level achieved by MOZ is the lowest due to the fact that efficient RB selection takes place which reduces the delays thus lowering levels of energy consumption. Also, only the optimal solution is selected which in turn results in energy gains and network lifetime.

In Figure 14, the memory consumption in bits versus the number of users is depicted. The DM takes decision based on the information it has and the solution depends on algorithms that run dynamically and this process is continuous until there are no more RBs to allocate. Moreover, it has been realized that the continuous assignment of RBs according to UE instances' needs utilizes less memory, which in turn increases network life time and performance and reduces delays.

3.2.6. $P R B$ Utilization. In this subsection we will show effect of the proposed algorithm on PRB utilization. The scenario depicts results according to two groups. In the first group, simulations are carried out without using the proposed MOZ; the second group implements MOZ. The performance is

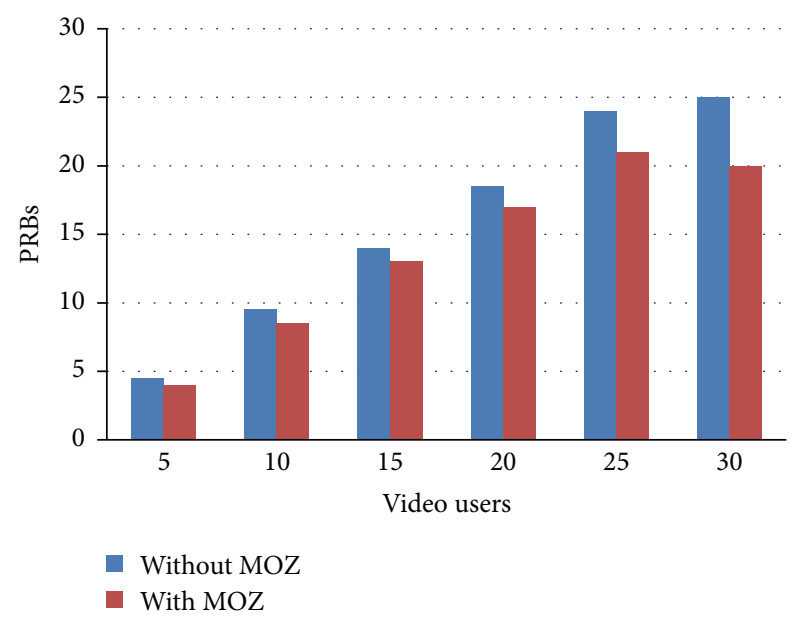

FIgURE 15: Mean PRBs used for video.

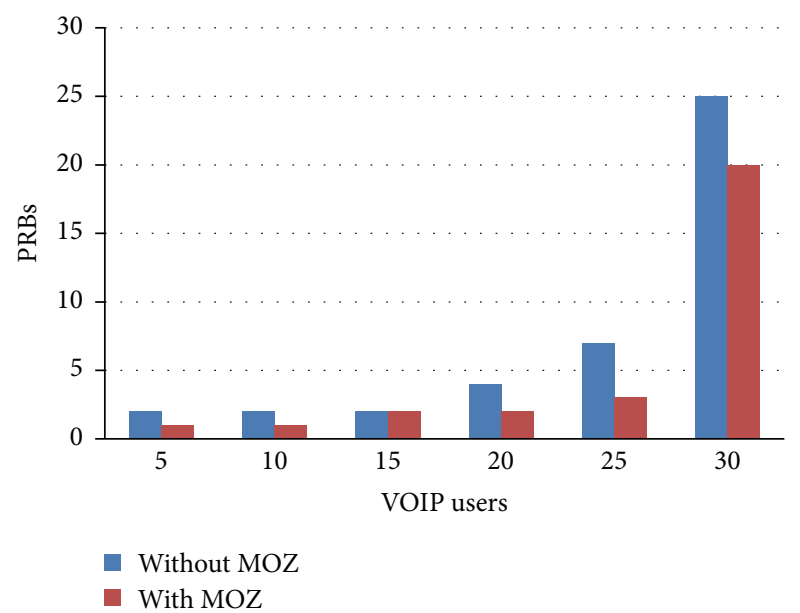

FIgURE 16: Mean PRBs used for VOIP.

measured in terms of mean PRBs being used for both video and VOIP users. In high load, the delay increases and a higher number of PRBs are used when we do not employ the MOZ algorithm. As a result after a specific limit no more requests can be fulfilled because all PRBs are utilized. Figure 15 depicts this scenario, as we can see that the average number of PRBs used with MOZ is less due to improved bandwidth utilization strategy, better coverage, and enhanced SNR. But with higher load of 30 UE instances, all the bandwidth is used up.

In Figure 16, for VOIP users also the average number of PRBs used is much less with increasing number of users.

3.3. Summary of the Simulation Analysis. Table 3 represents the results and statistics obtained by using MOZ where 1 to 4 represent the PO set. The optimal solution $O^{*}$ for each set of users is bold.

It can be clearly seen that the DM always selects the best feasible solution thus proving an accuracy of $100 \%$ in terms of optimal solution selection. The optimization procedure generates a PO set for varying number of users and out of this set the DM selects the best solution. 
TABLE 3: Statistics of the PO set and the optimal solution $\mathrm{O}^{*}$ selected using MOZ.

\begin{tabular}{lcccccccc}
\hline \multirow{2}{*}{ Number of users } & \multicolumn{3}{c}{ PO set (average delay in ms) } \\
\cline { 2 - 9 } & 1 & 2 & 3 & 4 & 1 & \multicolumn{3}{c}{ PO set (average throughput in bps) } \\
\hline 5 & 0.00252 & 0.00328 & $\mathbf{0 . 0 0 1 7 3}$ & 0.00175 & 407222.08000 & $\mathbf{6 6 4 1 8 7 . 3 8 6 6 6}$ & 501268.00000 & 394957.48000 \\
10 & $\mathbf{0 . 0 0 1 7 9}$ & 0.00197 & 0.00252 & 0.00328 & 15098.46667 & 28719.40000 & 43578.10667 & $\mathbf{5 8 5 8 0 . 8 6 6 6 7}$ \\
15 & $\mathbf{0 . 0 0 1 6 1}$ & 0.00167 & 0.00169 & 0.00175 & 408215.98667 & 762962.66667 & 930160.94667 & $\mathbf{9 3 3 4 6 3 . 9 7 3 3 3}$ \\
20 & 0.00161 & 0.00167 & 0.00159 & $\mathbf{0 . 0 0 1 5 5}$ & 408215.41334 & 766434.76000 & 973872.46667 & $\mathbf{1 0 1 5 9 6 7 . 7 8 6 6 7}$ \\
\hline
\end{tabular}

The evaluation and comparison of the simulation results show that MOZ performs very well for both voice and VOIP scenarios and performs better than the reference algorithm in terms of maximum achievable throughput and minimum delay. The reason behind these results is the use of optimization process employed by the DM which always selects the optimal solution for the arriving UE instances based on their QoS needs and priorities and channel quality. It further optimizes the procedure by maintaining queues to improve performance and achieves higher SNR and lower PLR. The cell spectral efficiency increases with each added UE that indicates improved system performance and is a proof of robust system design.

\section{Conclusions}

In this paper, we have proposed a multiobjective optimizer that helps in finding an optimal solution for selecting RBs for transmission based on the information provided to the decision maker. It provides an optimal solution for RRM to ensure QoS by incorporating the multiobjective optimization techniques that target to achieve higher throughput and lower delay. A set of Pareto optimal solutions is originated and is forwarded to the DM which chooses the best solution out of this set. We have adopted an interactive technique that embeds the multiobjective optimization with the modern techniques for RRM. Furthermore, the results are also provided that prove the efficiency of our proposed MOZ. This technique is innovative as it makes use of evolutionary MOO techniques (which are very powerful for finding optimal solution) and provides its implementation for LTE-A and will prove helpful in solving other issues for $4 \mathrm{G}$ and emerging $5 \mathrm{G}$ networks.

In future we aspire to investigate the opportunities for implementing MOO techniques for the evolving 5G networks that not only aim to deal with QoS issues but also target to achieve optimal energy consumption.

\section{Competing Interests}

The authors declare that there is no conflict of interests regarding the publication of this paper.

\section{References}

[1] S.-B. Lee, I. Pefkianakis, A. Meyerson, S. Xu, and S. Lu, "Proportional fair frequency-domain packet scheduling for 3GPP LTE uplink," in Proceedings of the 28th Conference on
Computer Communications (IEEE INFOCOM '09), pp. 26112615, Rio de Janeiro, Brazil, April 2009.

[2] "Physical layer aspects for evolved UTRA," 3GPP Technical Report 25.814, version 7.1.0, 2006.

[3] M. Nazir and F. Saleemi, "Cooperative cognitive ecology in selforganizing networks: a review article," International Journal of Computer Science and Information Security, vol. 10, no. 2, pp. 159-167, 2012.

[4] K. Deb, Multi-Objective Optimization with Evolutionary Algorithms, John Wiley \& Sons, New York, NY, USA, 1st edition, 2001.

[5] J. B. K. Deb and K. M. R. Słowinski, Multi-Objective Optimization: Interactive and Evolutionary Approaches, Springer, Berlin, Germany, 2008.

[6] A. De Domenico and E. C. Strinati, "A radio resource management scheduling algorithm for self-organizing femtocells," in Proceedings of the IEEE 21st International Symposium on Personal, Indoor and Mobile Radio Communications Workshops (PIMRC '10), pp. 191-196, IEEE, Instanbul, Turkey, September 2010.

[7] R. Kausar, Y. Chen, K. K. Chai, L. Cuthbert, and J. Schormans, "QoS aware mixed traffic packet scheduling in OFDMA-based LTE-advanced networks," in Proceedings of the 4th International Conference on Mobile Ubiquitous Computing, Systems, Services and Technologies (UBICOMM '10), pp. 53-58, Florence, France, October 2010.

[8] Y. J. Zhang and K. B. Letaief, "Adaptive resource allocation and scheduling for multiuser packet-based OFDM networks," in Proceedings of the IEEE International Conference on Communication, vol. 5, pp. 2949-2953, June 2004.

[9] C. Stocchi, N. Marchetti, and N. R. Prasad, "Self-optimized radio resource management techniques for LTE-a local area deployments," in Proceedings of the 2nd International Conference on Wireless Communication, Vehicular Technology, Information Theory and Aerospace \& Electronic Systems Technology (Wireless VITAE '11), pp. 1-5, Chennai, India, March 2011.

[10] M. Cenk Ertürk, H. Aki, I. Güvenç, and H. Arslan, "Fair and QoS-oriented spectrum splitting in macrocell-femtocell networks," in Proceedings of the 53rd IEEE Global Communications Conference (GLOBECOM '10), pp. 1-6, Miami, Fla, USA, December 2010.

[11] V. Chandrasekhar and J. G. Andrews, "Spectrum allocation in tiered cellular networks," IEEE Transactions on Communications, vol. 57, no. 10, pp. 3059-3068, 2009.

[12] A. Roy and S. K. Das, Optimizing QoS-Based Multicast Routing in Wireless Networks: A Multi-Objective Genetic Algorithmic Approach, Springer, New Yok, NY, USA, 2002.

[13] D. H. Lorenz and A. Orda, "QoS routing in networks with uncertain parameters," IEEE/ACM Transactions on Networking, vol. 6, no. 6, pp. 768-778, 1998. 
[14] A. M. Brintrup, J. Ramsden, H. Takagi, and A. Tiwari, "Ergonomic chair design by fusing qualitative and quantitative criteria using interactive genetic algorithms," IEEE Transactions on Evolutionary Computation, vol. 12, no. 3, pp. 343-354, 2008.

[15] R. Kamalian, H. Takagi, and A. Agogino, "Optimized design of MEMS by evolutionary multi-objective optimization with interactive evolutionary computation," in Genetic and Evolutionary Computation-GECCO 2004: Genetic and Evolutionary Computation Conference, Seattle, WA, USA, June 26-30, 2004. Proceedings, Part II, vol. 3103 of Lecture Notes in Computer Science, pp. 1030-1041, Springer, Berlin, Germany, 2004.

[16] S. Stefania, T. Issam, and B. Matthew, The UMTS Long Term Evolution Forum Theory to Practice, John Wiley \& Sons, New York, NY, USA, 2009.

[17] J. Puttonen, N. Kolehmainen, T. Henttonen, M. Moisio, and M. Rinne, "Mixed traffic packet scheduling in UTRAN long term evolution downlink," in Proceedings of the IEEE 19th International Symposium on Personal, Indoor and Mobile Radio Communications (PIMRC'08), pp. 1-5, Cannes, France, September 2008 .

[18] P. Won-Hyoung, C. Sunghyun, and B. Saewoong, "Scheduling design for multiple traffic classes in OFDMA networks," in Proceedings of the IEEE International Conference on Communications (ICC '06), pp. 790-795, Istanbul, Turky, 2006.

[19] T. Janevski, Traffic Analysis and Design of Wireless IP Networks, Artech House, Norwood, Mass, USA, 2003.

[20] K. Sandrasegaran, H. A. M. Ramli, and R. Basukala, "DelayPrioritized Scheduling (DPS) for real time traffic in 3GPP LTE system," in Proceedings of the IEEE Wireless Communications and Networking Conference (WCNC '10), pp. 1-6, IEEE, Sydney, Australia, April 2010.

[21] A. Machwe, I. C. Parmee, and J. C. Miles, "Multi-objective analysis of a component based representation within an interactive evolutionary design system," in Proceedings of the 7th International Conference in Adaptive Computing and Design and Manufacturing, 2006.

[22] K. C. Tan, T. H. Lee, D. Khoo, and E. F. Khor, "A multiobjective evolutionary algorithm toolbox for computer-aided multiobjective optimization," IEEE Transactions on Systems, Man, and Cybernetics, Part B: Cybernetics, vol. 31, no. 4, pp. 537-556, 2001.

[23] C. M. Fonseca and P. J. Fleming, "Multi-objective optimization and multiple constraint handling with evolutionary algorithms," IEEE Transactions on Systems, Man, and Cybernetics: Part A: Systems and Humans, vol. 28, no. 1, pp. 38-47, 1998.

[24] K. Miettinen, Nonlinear Multi-Objective Optimization, Kluwer, Boston, Mass, USA, 1999.

[25] V. Chankong and Y. Y. Haimes, Multi-Objective Decision Making Theory and Methodology, North-Holland, New York, NY, USA, 1983.

[26] K. Miettinen and M. M. Mäkelä, "Interactive bundle-based method for nondifferentiable multiobjective optimization: NIMBUS," Optimization, vol. 34, no. 3, pp. 231-246, 1995.

[27] F. Y. Edgeworth, Mathematical Psychics: An Essay on the Application of Mathematics to the Moral Sciences, C. Kegan Paul \& Co., London, UK, 1881.

[28] H. Kuhn and A. Tucker, "Nonlinear programming," in Proceedings of the Second Berkeley Symposium on Mathematical Statistics and Probability, J. Neyman, Ed., pp. 481-492, University of California Press, Berkeley, 1951.

[29] P. Korhonen, "Interactive methods," in Multiple Criteria Decision Analysis, J. Figueira, S. Greco, and M. Ehrgott, Eds., State of the Art Surveys, pp. 641-665, Springer, New York, NY, USA, 2005.

[30] F. Moety, S. Lahoud, B. Cousin, and K. Khawam, "A heuristic algorithm for joint power-delay minimization in green wireless access networks," in Proceedings of the International Conference on Computing, Networking and Communications (ICNC '15), pp. 280-286, IEEE, Garden Grove, Calif, USA, February 2015.

[31] S. Jun, N. Yi, A. Liu, and X. Haige, "Opportunistic scheduling for heterogeneous services in downlink OFDMA system," in Proceedings of the IEEE WRI International Conference on Communications and Mobile Computing( CMC '09), pp. 260264, Yunnan, China, January 2009.

[32] H. Jutler, "Linear model with several objective functions," Ekonomika I Matematiceckije Metody, vol. 3, pp. 397-406, 1967 (Polish).

[33] R. Solich, "Linear programming problem with several objective functions," Przeglad Statystyczny, vol. 16, pp. 24-30, 1969 (Polish).

[34] A. H. Ali, M. Nazir, A. Afzaal, and A. Sabah, "A traffic scheduler for radio resource management of Long Term EvolutionAdvanced (LTE-A)," Bahria University Journal of Information \& Communication Technologies, vol. 8, no. 1, 2015.

[35] G. Piro, L. A. Grieco, G. Boggia, F. Capozzi, and P. Camarda, "Simulating LTE cellular systems: an open-source framework," IEEE Transactions on Vehicular Technology, vol. 60, no. 2, pp. 498-513, 2011. 

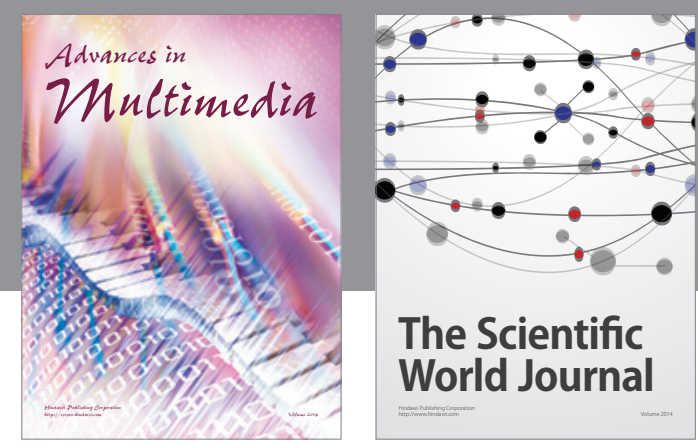

The Scientific World Journal
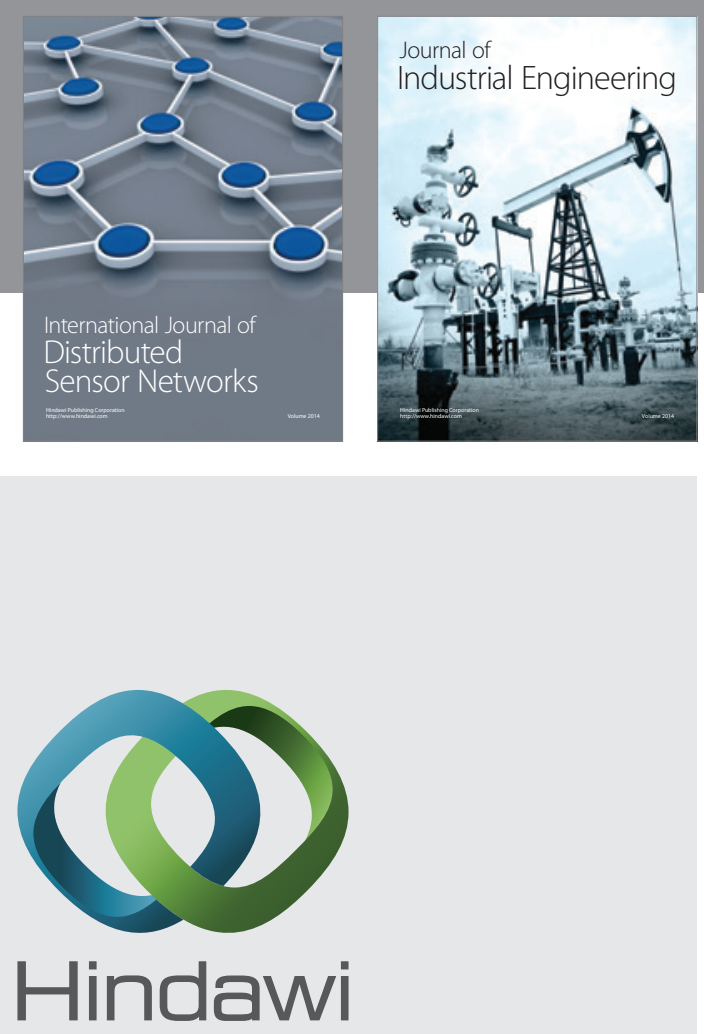

Submit your manuscripts at

http://www.hindawi.com

\section{Computer Networks} and Communications
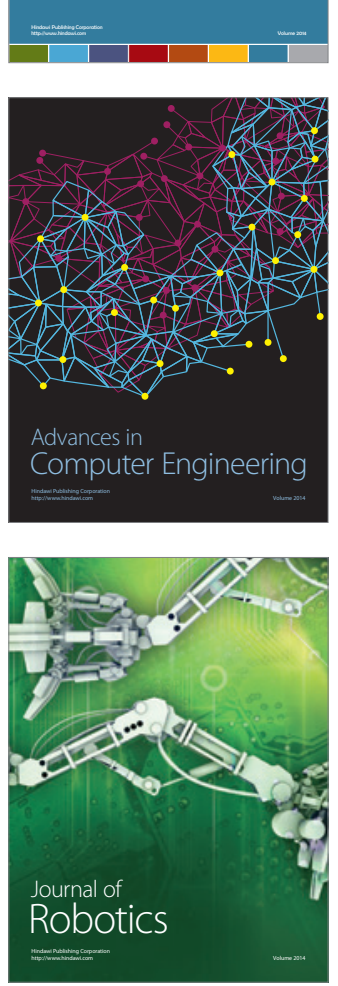
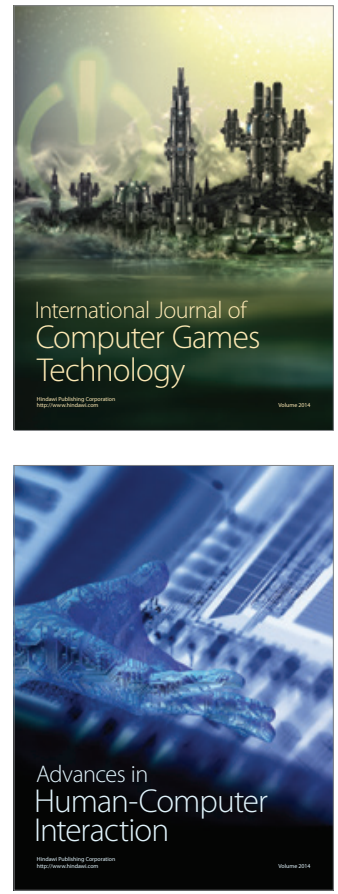
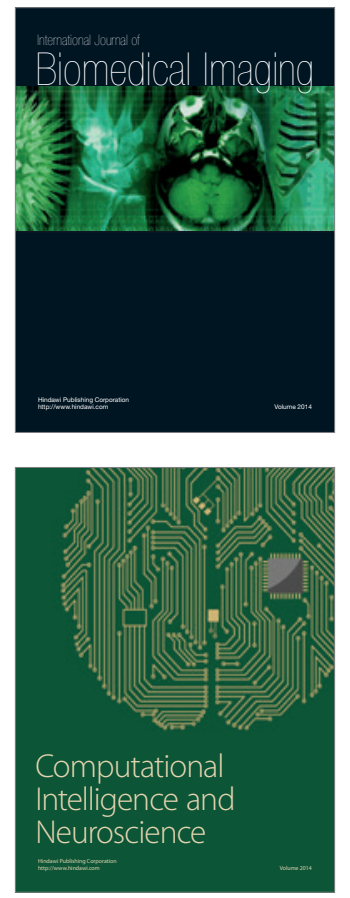
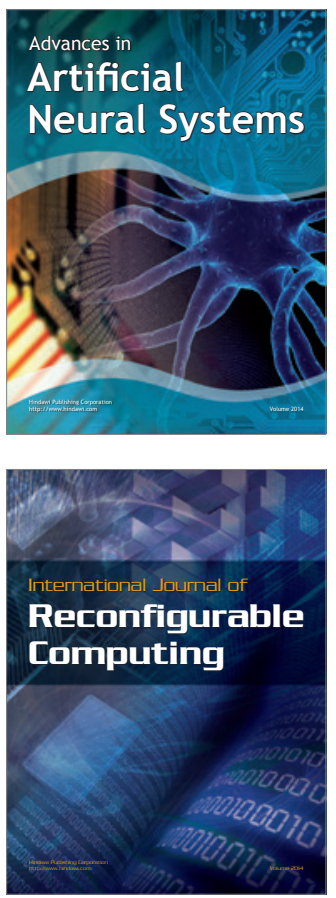
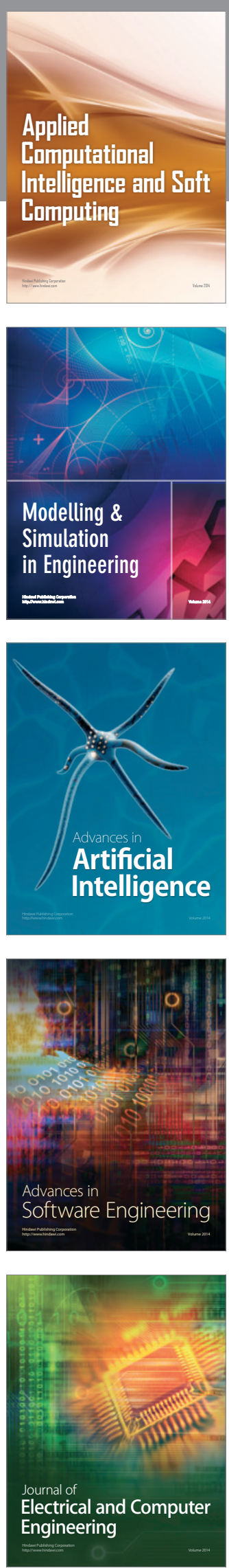